\title{
The relationship between snoring and left ventricular hypertrophy of China: a cross- sectional study
}

\author{
Naijin Zhang, Ning Ye, Yintao Chen, Xiaofan Guo, Guozhe Sun and Yingxian Sun *
}

\begin{abstract}
Background: Population-based investigations studying the association between snoring and left ventricular hypertrophy $(\mathrm{LVH})$ are lacking. Therefore, our study aims to investigate whether snoring is significantly associated with $L V H$, and to make clear the effect of varying degrees of snoring intensity on $L V H$.

Methods: A total of 10,139 participants were involved in this cross-sectional study. Snoring status and snoring intensity were evaluated by a structured questionnaire. LVH was defined as left ventricular mass index $\geq 51 \mathrm{~g} / \mathrm{m}^{2.7}$ for both men and women.

Results: The total prevalence of LVH was $10.0 \%$. the prevalence increased significantly in snorers according to snoring intensity, including low (10.3\%), normal (13.1\%), strong (14.7\%) and very strong (16.7 \%). After adjustment for age, race, gender, educational status, physical activity, annual income, current smoking status, current drinking status, sleep duration, hypertension, body mass index, waist circumference, low-density lipoprotein cholesterol, high-density lipoprotein cholesterol, triglyceride, total cholesterol and fasting plasma glucose, snoring (OR, 1.371; $95 \% \mathrm{Cl}, 1.147-1.637, P<0.001)$ was significantly associated with LVH. In addition, among the four kinds of intensity of snoring, normal $(\mathrm{OR}, 1.436 ; 95 \% \mathrm{Cl}$, 1.126-1.832, $P=0.004)$, strong $(\mathrm{OR}, 1.462 ; 95 \% \mathrm{Cl}, 1.124-1.902, P<0.001)$ and very strong $(\mathrm{OR}, 1.813 ; 95 \% \mathrm{Cl}, 1.273-2.684$, $P<0.001)$, rather than low $(\mathrm{OR}, 1.094 ; 95 \% \mathrm{Cl}, 0.834-1.434, P=0.518)$ were significantly associated with $\mathrm{LVH}$.
\end{abstract}

Conclusions: Snoring is independently associated with LVH. What's more, with the rise in snoring intensity, snoring will exert an increasing effect on LVH.

Keywords: Snoring, Snoring intensity, Left ventricular hypertrophy, Cardiovascular risk

\section{Background}

Snoring is a loud and jarring noise produced by the joint vibration of tongue and soft palate as a result of the obstructed breathing while sleeping. It is believed to be an important manifestation of obstructive sleep apnea (OSA). Exceptionally, however, still $40 \%$ male snorers and $20 \%$ female snorers do not experience OSA $[1,2]$. In addition, lots of studies in recent years showed that snoring was significantly associated with cardiovascular disease (CVD) [3-6].

There are many possible causes to CVD. Studies have verified that snoring is closely linked to metabolic syndrome $[7,8]$ which has always been considered as an

\footnotetext{
* Correspondence: yxsun@mail.cmu.edu.cn

Department of Cardiology, The First Hospital of China Medical University, 155 Nanjing North Street, Heping District, 110001 Shenyang, Liaoning, China
}

important independent risk factor of CVD $[9,10]$. Therefore, it is probable that metabolic syndrome mediates the connection between snoring and CVD. Atherosclerotic plaque is another probable way of mediating the connection between snoring and CVD, for recent studies have verified that snoring is closely associated with atherosclerosis $[11,12]$.

Having been long considered as an important risk factor of CVD [13, 14], left ventricular hypertrophy (LVH) is closely linked to myocardial infarction, stroke and arrhythmia [15]. Is there possibility that it is by mediating $\mathrm{LVH}$ that snoring results in CVD? As far as we know, there hasn't been any relevant studies to explore whether or not snoring is significantly associated with LVH in the general population. Besides, most previous studies, which estimated the effect of snoring on CVD, 
always focused on snoring frequency, but few studied the relationship between snoring and CVD by grading the snoring intensity. Hence, our study intend to ascertain whether snoring is significantly associated with $\mathrm{LVH}$, and to make clear the the effect of varying degrees of snoring intensity on LVH.

\section{Methods}

\section{Study population}

The study was conducted from January 2012 to August 2013. A representative sample from the participants aged over 35 years old was selected to present the prevalence, incidence and natural history of cardiovascular risk factors in rural areas of Liaoning Province. The study adopted a multi-stage, stratified, random-cluster sampling scheme. At the first stage, three areas of Dawa, Zhangwu and Liaoyang County were randomly selected from Liaoning province. At the second stage, one town was randomly selected from each county (for a total of three towns). At the third stage, 8 to 10 rural villages from each town were randomly selected (for a total of 26 rural villages). A total of 14,016 satisfactory participants were enrolled in this survey. Among those, 11,956 participants completed this study with a response rate of $85.3 \%$. And the participants who were pregnant or had malignant tumors or mental disorders or didn't know whether she/he snoring or missing variables for our multivariable logistic regression models were excluded. Finally, a sample size of 10,139 is accepted. The study was approved by the Ethics Committee of China Medical University, Shenyang, China and all procedures were conducted under ethical standards. Written consent was obtained from all participants after they had been informed of the objectives, benefits, medical items and confidentiality agreement regarding their personal information. For participants who were illiterate, written informed consent were obtained from their proxies.

\section{Echocardiography measurements}

Echocardiograms were performed on each participants by professional sonographers with a commercially available Doppler echocardiograph (Vivid, GE Healthcare, United States), using a $3.0-\mathrm{MHz}$ transducer. Subjects should maintain in the supine position and transthoracic echocardiogram included M-mode, 2-dimensional, spectral and color Doppler. Echocardiogram analyses were performed by three doctors specialized in echocardiography and consultations were made with two other doctors if questions or uncertainty arose. M-mode and two-dimensional images of the left ventricular (LV) wall thickness, internal diameter, aortic root and left atrium was being recorded by eath participants' parasternal acoustic window. Correct orientation of planes for Doppler recordings and imagings were verified according to previously described procedures [16, 17]. LV internal dimensions (LVID), posterior wall thickness (PWT) and interventricular septal thickness (IVST) were obtained according to American Society of Echocardiography recommendations $[17,18]$. Left ventricular mass was calculated according to the formula LVM [19] $=0.81$ (1.04 [LVID + IVST + PWT]) 3 - (LVID) $3+0.06$. According to de Simone et al, Left ventricular mass index (LVMI) was normalized for body height in $\mathrm{m}^{2.7}$ [20]. LVH was defined as the LVMI $\geq 51 \mathrm{~g} / \mathrm{m}^{2.7}$ for both men and women [20].

\section{Covariate measurements and definitions}

Information on covariates, such as demographic characteristics, lifestyle risk factors, family income and family history of chronic diseases, was collected during a single clinic visit by cardiologists and trained nurses using a standard questionnaire by face-to-face interview. Before the survey was performed, we invited all eligible investigators to attend the organized training. The training contents included the purpose of this study, how to administer the questionnaire, the standard method of measurement, the importance of standardization, and the study procedures. A strict test was evaluated after this training, only those who scored perfectly on the test could become investigators. During data collection, our inspectors had further instructions and support.

Race was categorized as Han or others which included some ethnic minorities in China, such as Mongol and Manchu. The questions posed for the educational level were categorized as primary school or below, middle school and high school or above. Sleep duration were self-reported from the participants by asking the following question, 'How many hours of sleep do you usually have everyday on average (including nocturnal sleep duration and nap duration)? Family income was categorized into three groups $55000,5000-20,000$ and $>20,000$ $\mathrm{CNY} /$ year. Current drinking status was defined as one or more alcoholic drinks in the previous year. Current smoking status was defined as a history of 100 or more cigarettes and continued use.

Physical activity which included occupational and leisure-time physical activity, was evaluated by a detailed description [21]. Occupational and leisure-time physical activity were merged and regrouped into 3 categories: 1) low was defined as participants who reported light levels of both occupational and leisuretime physical activity, 2) moderate was defined as participants who reported moderate or high levels of either occupational or leisure-time physical activity, 3) high was defined as participants who reported a moderate or high level of both occupational and leisure-time physical activity. 
According to American Heart Association protocol, blood pressure (BP) which was measured three times at two-min intervals after more than 10 min of rest, was measured by a standardized automatic electronic sphygmomanometer (HEM-907; Omron, Japan). Caffeinated beverages and exercise should be avoided for at least 30 min before the measurement. With the arm supported at the level of the heart, the participants were seated appropriately during the measurement. The mean of three blood pressure (BP) measures which was calculated accurately, was used in all analyses. And hypertension was defined as BP $\geq 140 / 90 \mathrm{mmHg}$ or currently taking hypertension medication, according to JNC-7 report guidelines [22].

Fasting blood samples were collected after $12 \mathrm{~h}$ of fasting in the morning. Blood samples were obtained from an antecubital vein into vacutainer tubes containing ethylenediaminetetraacetic acid (EDTA). Enzymatic reaction was used to analyze blood samples inculding fasting plasma glucose (FPG), high-density lipoprotein cholesterol (HDL-C), low-density lipoprotein cholesterol (LDL-C), triglyceride (TG), total cholesterol (TC) and other routine blood biochemical indexes on an Olympus AU640 autoanalyzer (Olympus, Kobe, Japan). All laboratory equipments were calibrated, and all blinded duplicate samples were used in our study.

\section{Information on snoring}

Snoring status was evaluated by a structured questionnaire, including two questions: 1 , Do you know, or someone tell you that you snore? (yes or no or don't know); and 2, How much is the loudness of sound when you are snoring? (Slightly louder than breathing sound $=1$; as loud as speaking $=2$; louder than normal speaking $=3$; so loud that it can be heard in the next room $=4$ ). According to the question 2, snoring intensity were categorized into low, normal, strong and very strong respectively. These responses were either self-reported or the response of a close relative and this method had been widely used to obtain the information of snoring, especially in epidemiologic studies $[3-6,11,12]$.

\section{Statistical Analysis}

Data were expressed as mean \pm standard deviation (SD) for continuous variables and numbers (percentages) for categorical variables. Differences in the characteristics of study participants between snorers and non-snorers were determined utilizing the t-test, ANOVA, nonparametric test or the $\mathrm{X}^{2}$-test, as appropriate. $\mathrm{LVH}$ was evaluated with multivariate logistic regression analyses according to snoring and its intensity. Adjustments for potential confounders were as follows: Model $1=$ unadjusted; Model 2 = adjusted for age, race, gender, educational status, physical activity, annual income, current smoking status, current drinking status and sleep duration; Model 3 = model 2 plus hypertension, waist circumference, low-density lipoprotein cholesterol, high-density lipoprotein cholesterol, triglyceride, total cholesterol and fasting plasma glucose. Results of logistic regression analyses are reported as odds ratios (ORs) and corresponding $95 \%$ confidence intervals (CIs). All the statistical analyses were calculated using programs available in the SPSS version 22.0 software (IBM Corp., Armonk, NY, USA), and P values were considered to be statistically significant if less than 0.05 .

\section{Results}

Baseline characteristics of participants between snorers and non-snorers

A total of 10,139 participants were involved in this cross-sectional study. the prevalence of snoring was $42.2 \%(n=4275)$ and the prevalence of four kinds of snoring intensity including low, normal, strong and very strong was $13.9 \%(n=1412), 14.3 \%(n=1450), 10.4 \%$ $(n=1059)$ and $3.5 \%(n=354)$ respectively (Fig. 1$)$. Compared with non-snorers, snorers were significantly older and had higher levels of systolic blood pressure, diastolic blood pressure, body mass index, height, waist circumference, low-density lipoprotein cholesterol, triglyceride, total cholesterol and the prevalence of current smoking, drinking, diabetes and hypertension (Table 1) (all Ps $<0.05$ ). However, there was no significant difference between the

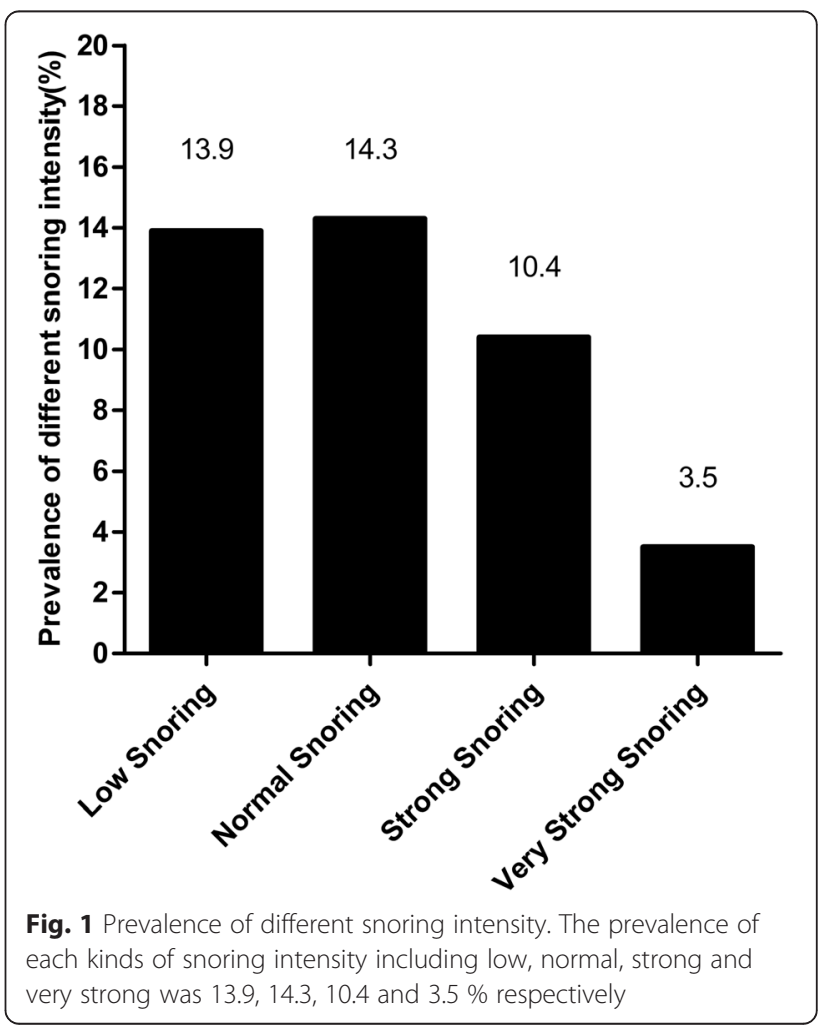


Table 1 Characteristics of participants according to snoring status

\begin{tabular}{|c|c|c|c|}
\hline \multirow{2}{*}{$\begin{array}{l}\text { Variables } \\
\text { Mean } \pm \text { SD }\end{array}$} & \multirow[t]{2}{*}{ Non-snorers } & \multirow[t]{2}{*}{ Snorers } & \multirow[t]{2}{*}{$P$-value } \\
\hline & & & \\
\hline Age (year) & $53.3 \pm 10.9$ & $54.5 \pm 9.9$ & $<0.001$ \\
\hline Male gender (\%) & $2475(42.2)$ & $2216(51.8)$ & $<0.001$ \\
\hline Race (Han) (\%) & $5572(95.0)$ & $4067(95.1)$ & 0.793 \\
\hline Educational status (\%) & & & 0.065 \\
\hline Primary school or below & $2882(49.1)$ & $2182(51.0)$ & \\
\hline Middle school & $2448(41.7)$ & $1686(39.4)$ & \\
\hline High school or above & $534(9.1)$ & $407(9.5)$ & \\
\hline Physical activity (\%) & & & 0.102 \\
\hline Light & $1699(29.0)$ & $1261(29.5)$ & \\
\hline Moderate & $3852(65.7)$ & $2747(64.3)$ & \\
\hline Severe & $313(5.3)$ & $267(6.2)$ & \\
\hline Annual income (CNY/year) & & & 0.662 \\
\hline$\leq 5000$ & $732(12.5)$ & $520(12.2)$ & \\
\hline $5000-20000$ & $3161(53.9)$ & $2343(54.8)$ & \\
\hline$>20000$ & 1971 (33.6) & $1412(33.0)$ & \\
\hline Current smoking status (\%) & $1916(32.7)$ & $1672(39.1)$ & $<0.001$ \\
\hline Current drinking status (\%) & $1155(19.7)$ & 1139 (26.6) & $<0.001$ \\
\hline Sleep duration (h/d) & $7.2 \pm 1.7$ & $7.3 \pm 1.7$ & 0.069 \\
\hline Systolic blood pressure (mmHg) & $138.9 \pm 22.8$ & $145.0 \pm 23.3$ & $<0.001$ \\
\hline Diastolic blood pressure $(\mathrm{mmHg})$ & $80.6 \pm 11.2$ & $83.8 \pm 12.0$ & $<0.001$ \\
\hline Height (cm) & $160.5 \pm 8.0$ & $161.4 \pm 8.3$ & $<0.001$ \\
\hline Body mass index (kg/m2) & $24.1 \pm 3.4$ & $25.7 \pm 3.8$ & $<0.001$ \\
\hline Waist circumference (cm) & $80.3 \pm 9.3$ & $85.2 \pm 9.9$ & $<0.001$ \\
\hline Low-density lipoprotein cholesterol (mmol/L) & $2.9 \pm 0.8$ & $3.0 \pm 0.8$ & $<0.001$ \\
\hline High-density lipoprotein cholesterol (mmol/L) & $1.4 \pm 0.4$ & $1.4 \pm 0.4$ & $<0.001$ \\
\hline Triglyceride (mmol/L) & $1.5 \pm 1.4$ & $1.8 \pm 1.6$ & $<0.001$ \\
\hline Total cholesterol (mmol/L) & $5.2 \pm 1.1$ & $5.3 \pm 1.1$ & $<0.001$ \\
\hline Fasting plasma glucose (mmol/L) & $5.8 \pm 1.5$ & $6.0 \pm 1.7$ & $<0.001$ \\
\hline Diabetes (\%) & $503(8.6)$ & $533(12.5)$ & $<0.001$ \\
\hline Hypertension (\%) & $2648(45.2)$ & $2482(58.1)$ & $<0.001$ \\
\hline
\end{tabular}

two groups in race, sleep duration and levels of educational status, physical activity and annual income.

\section{Echocardiographic characteristics of participants according to snoring status}

As the results showed in Table 2, compared with nonsnorers, snorers had significantly higher levels of LVID, IVST, PWT, left atrial dimension (LAD), left ventricular mass, left ventricular mass index and prevalence of left ventricular hypertrophy, and lower levels of early/late diastolic peak flow velocity (E/A) (all Ps $<0.05$ ). However, there was no significant difference between the two groups in RWT and ejection fraction (EF).
The prevalence of LVH by different grades of the snoring intensity

The total prevalence of LVH was $10.0 \%$ and the prevalence of LVH in non-snorers was $7.9 \%$. The prevalence increased significantly in snorers according to snoring intensity, including low (10.3\%), normal (13.1\%), strong (14.7\%) and very strong (16.7 \%) (Fig. 2).

In Table 3, we estimated the ORs between snoring and LVH. Unadjusted in model 1, snoring (OR, 1.734; $95 \%$ CI, 1.522-1.976, $P<0.001)$ was significantly associated with $\mathrm{LVH}$. Among the four kinds of intensity of snoring, low (OR, 1.352; 95 \% CI, 1.111-1.644, $P=0.003$ ), normal (OR, 1.767; $95 \% \mathrm{CI}, 1.477-2.115, P<0.001)$, strong 
Table 2 Echocardiographic characteristics of participants according to snoring status

\begin{tabular}{|c|c|c|c|}
\hline Variables & Non-snorers & Snorers & $P$-value \\
\hline \multicolumn{4}{|l|}{ Mean \pm SD } \\
\hline End-diastolic left ventricular internal dimension (LVID), mm & $4.7 \pm 0.4$ & $4.8 \pm 0.4$ & $<0.001$ \\
\hline End-diastolic interventricular septum thickness (IVST), mm & $0.9 \pm 0.3$ & $1.0 \pm 0.2$ & $<0.001$ \\
\hline End-diastolic posterior wall thickness (PWT), mm & $0.9 \pm 0.3$ & $1.0 \pm 0.2$ & $<0.001$ \\
\hline Myocardial relative wall thickness (RWT), mm & $0.4 \pm 0.1$ & $0.4 \pm 0.1$ & 0.074 \\
\hline Left atrial dimension (LAD) (mm) & $3.3 \pm 0.4$ & $3.4 \pm 0.4$ & $<0.001$ \\
\hline Ejection fraction (EF), (\%) & $63.0 \pm 3.8$ & $62.9 \pm 3.8$ & 0.192 \\
\hline Early/late diastolic peak flow velocity (E/A) ratio & $1.1 \pm 0.5$ & $1.0 \pm 1.5$ & 0.012 \\
\hline Left ventricular mass $(\mathrm{g})$ & $137.8 \pm 98.3$ & $149.1 \pm 80.0$ & $<0.001$ \\
\hline Left ventricular mass index $\left(\mathrm{g} / \mathrm{m}^{2.7}\right)$ & $38.8 \pm 27.6$ & $41.0 \pm 20.7$ & $<0.001$ \\
\hline Prevalence of left ventricular hypertrophy, \% & $461(7.9)$ & 551 (12.9) & $<0.001$ \\
\hline
\end{tabular}

(OR, 2.025; $95 \% \mathrm{CI}, 1.666-2.460, P<0.001)$ and very strong (OR, 2.344; $95 \%$ CI, 1.745-3.149, $P<0.001$ ) were all significantly associated with LVH. After adjustment for age, race, gender, educational status, physical activity, annual income, current smoking status, current drinking status and sleep duration in model 2, snoring (OR, 1.709; $95 \%$ CI, 1.495-1.953, $P<0.001$ ) was significantly associated with LVH. In addition, among the four kinds of intensity of snoring, low (OR, 1.381; $95 \%$ CI, 1.131-1.684, $P=0.002)$, normal (OR, 1.774; $95 \% \mathrm{CI}, 1.476-2.133$, $P<0.001$ ), strong (OR, 1.886; $95 \% \mathrm{CI}, 1.545-2.302$, $P<0.001)$ and very strong (OR, 2.199; $95 \% \mathrm{CI}, 1.624-$ 2.977, $P<0.001$ ) were all significantly associated with LVH. To farther ascertain the role that metabolic factors play in this relationship, we adjusted model 2 and introduced hypertension, body mass index, waist circumference, low-density lipoprotein cholesterol, high-density lipoprotein cholesterol, triglyceride, total cholesterol and fasting plasma glucose into model 3. Interestingly, still the increased ORs could be observed in group snoring (OR, 1.371; $95 \% \mathrm{CI}, 1.147-1.637, P<0.001$ ), normal (OR, 1.436; $95 \% \mathrm{CI}, 1.126-1.832, P=0.004)$, strong (OR, 1.462; $95 \% \mathrm{CI}, 1.124-1.902, P<0.001)$ and very strong $(\mathrm{OR}, 1.813$; $95 \% \mathrm{CI}, 1.273-2.684, P<0.001)$ intensity of snoring, rather than low (OR, 1.094; $95 \% \mathrm{CI}$, $0.834-1.434, P=0.518$ ) intensity of snoring. But the ORs were attenuated when we further adjusted for these metabolic factors.

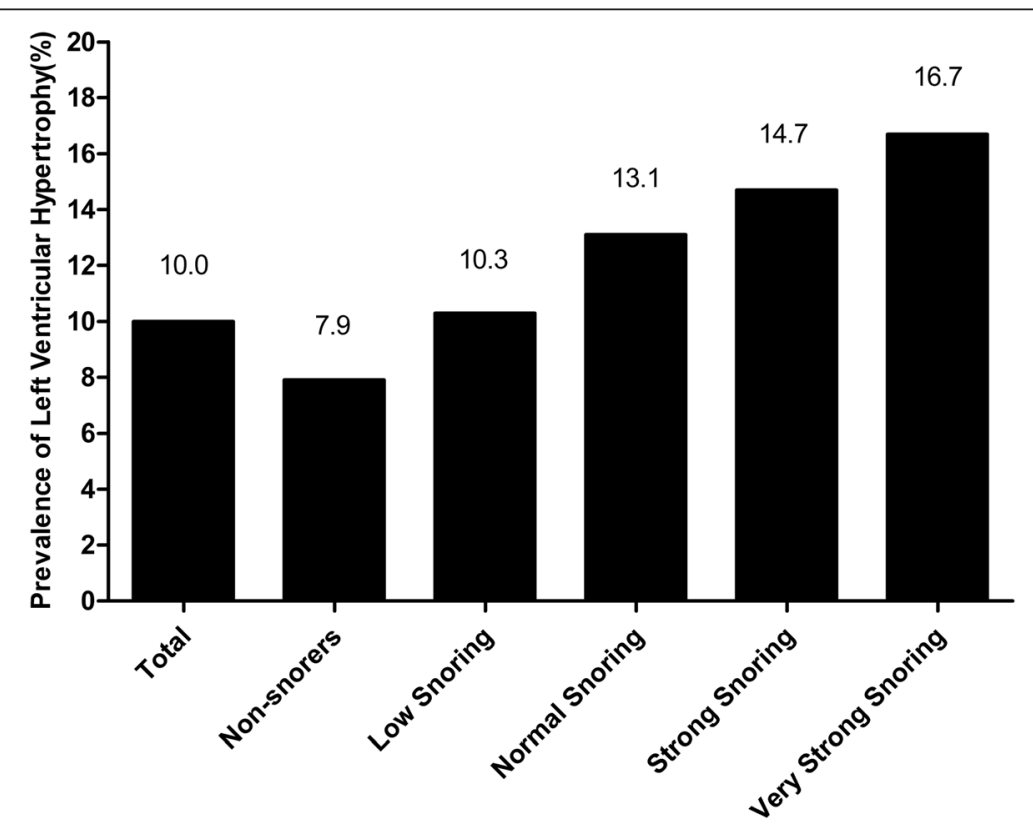

Fig. 2 Prevalence of left ventricular hypertrophy by different grades of the snoring intensity. The total prevalence of LVH was $10.0 \%$ and the prevalence of LVH in non-snorers was $7.9 \%$. The prevalence increased significantly in snorers according to snoring intensity, including low (10.3\%), normal (13.1\%), strong (14.7\%) and very strong (16.7\%). Abbreviations: LVH = left ventricular hypertrophy 
Table 3 Multiple regression analyses for the relationship between snoring status, snoring intensity and left ventricular hypertrophy

\begin{tabular}{|c|c|c|c|c|c|c|}
\hline & \multicolumn{2}{|l|}{ Model 1} & \multicolumn{2}{|l|}{ Model 2} & \multicolumn{2}{|l|}{ Model 3} \\
\hline & OR $(95 \%$ Cl) & $P$ & OR (95 \% Cl) & $P$ & OR $(95 \% \mathrm{Cl})$ & $P$ \\
\hline \multicolumn{7}{|l|}{ Snoring status } \\
\hline Non-snorers & 1.000 (reference) & & 1.000 (reference) & & 1.000 (reference) & \\
\hline Snorers & $1.734(1.522-1.976)$ & $<0.001$ & 1.709 (1.495-1.953) & $<0.001$ & $1.371(1.147-1.637)$ & $<0.001$ \\
\hline \multicolumn{7}{|c|}{ Snoring intensity } \\
\hline Non-snorers & 1.000 (reference) & & 1.000 (reference) & & 1.000 (reference) & \\
\hline Low & $1.352(1.111-1.644)$ & 0.003 & $1.381(1.131-1.687)$ & 0.002 & $1.094(0.834-1.434)$ & 0.518 \\
\hline Normal & $1.767(1.477-2.115)$ & $<0.001$ & $1.774(1.476-2.133)$ & $<0.001$ & $1.436(1.126-1.832)$ & 0.004 \\
\hline Strong & $2.025(1.666-2.460)$ & $<0.001$ & $1.886(1.545-2.302)$ & $<0.001$ & $1.462(1.124-1.902)$ & $<0.001$ \\
\hline Very strong & $2.344(1.745-3.149)$ & $<0.001$ & 2.199 (1.624-2.977) & $<0.001$ & $1.813(1.273-2.684)$ & $<0.001$ \\
\hline
\end{tabular}

Model 1: unadjusted

Model 2: adjusted for age, race, gender, educational status, physical activity, annual income, current smoking status, current drinking status and sleep duration Model 3: adjusted for model 1 plus hypertension, body mass index, waist circumference, low-density lipoprotein cholesterol, high-density lipoprotein cholesterol, triglyceride, total cholesterol and fasting plasma glucose

$\mathrm{Cl}$ confidence interval, OR Odds ratio

\section{Discussion}

The main findings of this large-scale cross-section study were as follows: Firstly, after adjustment for age, race, gender, educational status, physical activity, annual income, current smoking status, current drinking status and sleep duration, snoring and four kinds of snoring intensity were significantly associated with LVH. Secondly, when we further adjusted for the metabolic factors, snoring, normal, strong and very strong snoring intensity, but not low snoring intensity, were significantly associated with LVH. However, the ORs which evaluate the association between snoring, snoring intensity and LVH were attenuated. This suggests that metabolic factors partly mediate the association between snoring, snoring intensity and LVH. Finally, with the increase in snoring intensity, snoring was increasingly risky to $\mathrm{LVH}$. Also we found that the prevalence of $\mathrm{LVH}$ increased significantly with the rise in snoring intensity.

Epidemiologic studies have showed that snoring is significantly associated with the development of CVD [3-6]. However, As far as we know, this is the first study to show that snoring is signifacantly associated with $\mathrm{LVH}$ in the general population, and also the first to probe into the effect of varying degrees of snoring intensity on CVD. The biological mechanisms that link snoring to LVH remain to be fully elucidated, but some of mechanisms have been proposed. we know that some of the snorers are often accompanied by OSA. It has been verified in relevant studies that OSA constitutes an important risk factor of ventricular remodeling $[23,24]$. OSA can reduce the oxygen saturation indices and cause acute sympathetic activation, hemodynamic change and platelet aggregation, which increase cardiac output and induce ventricular remodeling. This is one of the reasons why snoring can lead to LVH. Previous studies showed particular interest in the relationship between OSA and ventricular remodeling; however, the detection of OSA has to use polysomnography. As a result, only a handful of subjects were involved in the studies, far from being persuasive. Compared with OSA, snoring can be used for questionnaire survey to collect data and suitable for large-scale epidemiological investigations [3-6]. In addition, snorers capture snoring symptoms experienced over time, which cannot be acquired by overnight measurement [11].

Metabolic disorder is another probable way of mediating the association between snoring and LVH. In the present study, compared with non-snorers, snorers appear to be more obese, hypertensive, hyperlipemia and diabetic, and the ORs which evaluate the association between snoring, snoring intensity and LVH were attenuated when we further adjusted for these metabolic factors. This suggests that metabolic disorders partly mediate the association between snoring and LVH. As is known to us that hypertension ranks among the most important risk factors of LVH [25]. What's more, it has been reported in relevant studies that snoring serves as an important independent risk factor of hypertension $[26,27]$. Liang Sun [7] showed that snoring, independent of lifestyle factors, adiposity, inflammatory markers and adipokines in apparently healthy Chinese, was significantly associated with metabolic syndrome. And it has been verified in numerous studies that metabolic syndrome is an important risk factor of LVH. Mats Halldin proved in his study [28] that high blood pressure and WC were independent risk factors for LVH and argued 
that the LVH might result from the changes in the mediated IGF-1 and IGF-Binding Protein-1. Halldin M [29] proved that it was high blood pressure, abdominal obesity and high glucose levels (only limited to in females) that caused LVH. In view of this, we propose those who have experienced chronic snoring should be careful about LVH. Besides, while treating LVH patients with severe snoring, physicians should give appropriate treatment to the snoring of patients.

Nevertheless, there still remains some limitations in our study. Firstly, as our study is based on crosssectional designs, it is unable to distinguish between cause and effect. Therefore, we propose to verify our conclusions through relevant cohort studies. Secondly, our data on snoring and snoring intensity were obtained from self-report questionnaires, and some subjects living alone were likely to be unaware of their snoring status. Misclassification of snoring status might have attenuated the connection between snoring and LVH, though those who were unclear of their snoring conditions had been excluded from the questionnaires. Finally, our study aims only at Chinese populations. As a result, the present results may not be applicable to populations from other regions and ethnic groups.

\section{Conclusion}

this large-scale cross-sectional study shows that snoring is independently associated with LVH. What's more, with the rise in snoring intensity, snoring will exert an increasing effect on LVH.

\begin{abstract}
Abbreviations
LVH: left ventricular hypertrophy; CVD: Cardiovascular disease; BMI: Body mass index; MetS: metabolic syndrome; WC: Waist circumference; BP: Blood pressure; FPG: Fasting plasma glucose; TC: Total cholesterol; LDL-C: Lowdensity lipoprotein cholesterol; HDL-C: High-density lipoprotein cholesterol; TG: Triglyceride; LVID: end-diastolic left ventricular internal dimension; IVST: end-diastolic interventricular septum thickness; PWT: end-diastolic posterior wall thickness; RWT: myocardial relative wall thickness; LAD: left atrial dimension; EF: ejection fraction; E/A: early/late diastolic peak flow velocity; LVM: left ventricular mass; LVMI: left ventricular mass index; EDTA: ethylenediaminetetraacetic acid; SBP: Systolic BP; DBP: Diastolic BP; WHO: World Health Organization; OR: Odds ratio; Cl: Confidence interval.
\end{abstract}

\section{Competing interests}

The authors declare that they have no competing interests.

\section{Authors' contributions}

NZ participated in the design, coordination and execution of the study, analysis and interpretation of data, writing of the manuscript. YS contributed to the study design, interpretation of data and research team. XG, GS, YC, NY participated in supervision of the project, contributed to critical revision of the manuscript. All authors read and approved the final manuscript.

\section{Acknowledgements}

This study was supported by grants from the "Twelfth Five-Year" project funds (National Science and Technology Support Program of China, Grant \#2012BAJ18B02) that Pro Yingxian Sun responsible for enable the project completion.

Received: 9 October 2015 Accepted: 7 January 2016

Published online: 15 January 2016

\section{References}

1. Bearpark H, Elliott L, Grunstein R, Cullen S, Schneider H, Althaus W, et al. Snoring and sleep apnea: A population study in Australian men. Am J Respir Crit Care Med. 1995;151(5):1459-65.

2. Ohayon MM, Guilleminault C, Priest RG, Caulet M. Snoring and breathing pauses during sleep: telephone interview survey of a United Kingdom population sample. BMJ. 1997;314:860-3.

3. Nagayoshi M, Tanigawa T, Yamagishi K, Sakurai S, Kitamura A, Kiyama M, et al. Self-Reported Snoring Frequency and Incidence of Cardiovascular Disease: The Circulatory Risk in Communities Study (CIRCS). J Epidemiol. 2012;22:295-301.

4. Hu FB, Willett WC, Manson JE, Colditz GA, Rimm EB, Speizer FE, et al. Snoring and risk of cardiovascular disease in women. J Am Coll Cardiol. 2000;35(2):308-13.

5. Janszky I, Ljung R, Rohani M, Hallqvist J. Heavy snoring is a risk factor for case fatality and poor short-term prognosis after a first acute myocardial infarction. Sleep. 2008;31(6):801-7.

6. Zamarrón C, Gude F, Otero Otero Y, Rodríguez-Suárez JR. Snoring and myocardial infarction: a 4-year follow-up study. Respir Med. 1999;93:108-12.

7. Sun L, Pan A, Yu Z, Li H, Shi A, Yu D, et al. Snoring, inflammatory markers, adipokines and metabolic syndrome in apparently healthy Chinese. PLoS One. 2011;6(11):e27515.

8. Roopa M, Deepa M, Indulekha K, Mohan V. Prevalence of sleep abnormalities and their association with metabolic syndrome among Asian Indians: Chennai Urban Rural Epidemiology Study (CURES-67). J Diabetes Sci Technol. 2010;4(6):1524-31.

9. Sundström J, Arnlöv J, Stolare K, Lind L. Blood-pressure-independent relations of left ventricular geometry to the metabolic syndrome: a population-study. Heart. 2010;94:874-8.

10. Guerra F, Mancinelli L, Angelini L, Fortunati M, Rappelli A, Dessì-Fulgheri P, et al. The association of left ventricular hypertrophy with metabolic syndrome is dependent on body mass index in hypertensive overweight or obese patients. PLoS One. 2011;6(1):e16630.

11. Li Y, Liu J, Wang W, Yong Q, Zhou G, Wang M, et al. Association of selfreported snoring with carotid artery intimamedia thickness and plaque. J Sleep Res. 2012;21:87-93.

12. Lee Y-H, Kweon S-S, Choi BY, Kim MK, Chun B-Y, Shin DH, et al. Selfreported Snoring and Carotid Atherosclerosis in Middle-aged and Older Adults: The Korean Multi-Rural Communities Cohort Study. J Epidemiol. 2014:24(4):281-6.

13. Bluemke DA, Kronmal RA, Lima JA, Liu K, Olson J, Burke GL, et al. The relationship of left ventricular mass and geometry to incident cardiovascular events: the MESA (Multi-Ethnic Study of Atherosclerosis) study. J Am Coll Cardiol. 2008:52:2148-55.

14. Akili BA, Okin PM, Devereux RB. Prognostic implications of left ventricular hypertrophy. Am Heart J. 2001;141(3):334-41.

15. Koren MJ, Devereux RB, Casale PN, Savage DD, Laragh JH. Relation of left ventricular mass and geometry to morbidity and mortality in uncomplicated essential hypertension. Ann Intern Med. 1991;114:345-52.

16. Devereux RB, Roman MJ. Evaluation of cardiac and vascular structure by echocardiography and other noninvasive techniques. In: Hypertension: Pathophysiology, Diagnosis, Treatment. 2nd ed. New York: Raven; 1995. p. 1969-85.

17. Sahn DJ, DeMaria A, Kisslo J, Weyman A. Recommendations regarding quantitation in M-mode echocardiography: results of a survey of echocardiographic measurements. Circulation. 1978;58(6):1072-83.

18. Schiller NB, Shah PM, Crawford M, DeMaria A, Devereux R, Feigenbaum H, et al. Recommendations for quantitation of the left ventricle by twodimensional echocardiography. American Society of Echocardiography Committee on Standards, Subcommittee on Quantitation of TwoDimensional Echocardiograms. J Am Soc Echocardiogr. 1989;2(5):358-67.

19. Devereux RB, Alonso DR, Lutas EM, Gottlieb GJ, Campo E, Sachs I, et al. Echocardiographic assessment of left ventricular hypertrophy: comparison to necropsy findings. Am J Cardiol. 1986;57(6):450-8.

20. de Simone G, Izzo R, De Luca N, Gerdts E. Left ventricular geometry in obesity: is it what we expect? Nutr Metab Cardiovasc Dis. 2013; 23(10):905-12.

21. Hu G, Tuomilehto J, Silventoinen $K$, Barengo $N$, Jousilahti P. Joint effects of physical activity, body mass index, waist circumference and waist-to-hip ratio with the risk of cardiovascular disease among middle-aged Finnish men and women. Eur Heart J. 2004;25(24):2212-9. 
22. Chobanian AV, Bakris GL, Black HR, Cushman WC, Green LA, Izzo Jr JL, et al. Seventh report of the Joint National Committee on Prevention, Detection, Evaluation, and Treatment of High Blood Pressure. Hypertension. 2003;42(6):1206-52.

23. Koga S, Ikeda S, Nakata T, Yasunaga T, Maemura K. Effects of nasal continuous positive airway pressure on left ventricular concentric hypertrophy in obstructive sleep apnea syndrome. Intern Med. 2012;51(20):2863-8.

24. Prejbisz A, Florczak E, Pręgowska-Chwała B, Klisiewicz A, KuśmierczykDroszcz B, Zieliński T, et al. Relationship between obstructive sleep apnea and markers of cardiovascular alterations in never-treated hypertensive patients. Hypertens Res. 2014;37(6):573-9.

25. Silva EA, Flexa F, Zanella MT. Abdominal obesity, insulin resistance and hypertension: impact on left ventricular mass and function in women. Arq Bras Cardiol. 2007;89(2):77-82. 86-92.

26. Wang T, Lu J, Wang W, Mu Y, Zhao J, Liu C, et al. Sleep duration and snoring associate with hypertension and glycaemic control in patients with diabetes. Diabet Med. 2015;32(8):1001-7.

27. Davies RJ, Crosby J, Prothero A, Stradling JR. Ambulatory blood pressure and left ventricular hypertrophy in subjects with untreated obstructive sleep apnoea and snoring, compared with matched control subjects, and their response to treatment. Clin Sci (Lond). 1994;86(4):417-24.

28. Halldin M, Brismar $K$, Fahlstadius $P$, Vikström M, de Faire U, Hellénius ML. The Metabolic Syndrome and ECG Detected Left Ventricular Hypertrophy Influences from IGF-1 and IGF-Binding Protein-1. PLoS One. 2014;9(12): e108872.

29. Halldin M, Fahlstadius P, de Faire U, Vikström M, Hellénius ML. The metabolic syndrome and left ventricular hypertrophy-the influence of gender and physical activity. Blood Press. 2012;21(3):153-60.

\section{Submit your next manuscript to BioMed Central and we will help you at every step:}

- We accept pre-submission inquiries

- Our selector tool helps you to find the most relevant journal

- We provide round the clock customer support

- Convenient online submission

- Thorough peer review

- Inclusion in PubMed and all major indexing services

- Maximum visibility for your research

Submit your manuscript at www.biomedcentral.com/submit
Biomed Central 